Check for updates

Cite this: RSC Adv., 2017, 7, 22531

Received 16th March 2017

Accepted 14th April 2017

DOI: 10.1039/c7ra03121a

rsc.li/rsc-advances

\title{
In situ ozonolysis of polypropylene during extrusion to produce long-chain branches with the aid of TMPTA
}

\author{
Guang-Jian He, (D) ${ }^{a}$ Bing-Yu Yuan, ${ }^{a}$ Ting-Ting Zheng, ${ }^{a}$ Wen-li Zhu ${ }^{b}$ \\ and Xiao-Chun Yin*a
}

\begin{abstract}
The introduction of long-chain branches (LCBs) in polypropylene (PP) during the extrusion process is normally induced by peroxide chemicals which are known to lead to the formation of secondary products in the resin. Here we report a novel synthesis method to prepare LCB-PP via in situ ozonolysis during reactive extrusion in the presence of a multifunctional agent. Depending on the fast ozonation of molten PP molecules, free radicals can be generated in PP macromolecules and induce chain scission reactions during extrusion. LCB structures of PP could be formed when a multifunctional agent, trimethylolpropane triacrylate (TMPTA), was added into the PP matrix during extrusion. The effect of reaction temperature, polymer flow rate and TMPTA concentration on the LCB-PP structures is discussed. Molecular parameters of LCB-PP were detected by MALS SEC technique. Various rheological plots including elongation rheological properties were applied to distinguish the LCB structures in PP samples. Without residues and by-products of peroxide in the final polymer resin, this synthesis method of LCB-PP has highly efficient and easily adjustable merits.
\end{abstract}

\section{Introduction}

Compared to other thermoplastics, the commodity resin polypropylene (PP) has many advantageous properties, such as high tensile modulus, excellent chemical resistance, low density, low cost and easy processing. However, because of its highly linear chains and a relatively narrow molar mass distribution, PP exhibits low melt strength and weak strain hardening behavior which limits its applications in foaming, thermoforming, extrusion coating, and blow molding processes in which the type of flow is predominantly elongation flow. It has been reported that the most efficient way to enhance the melt strength of linear PP is by introducing long chain branches (LCB) to the main chains of linear PP, and even a small amount of long chain branches could have a significant effect on the melt strength, rheological, thermal and mechanical properties. ${ }^{1-5}$

LCB-PP could be synthesized by direct polymerization ${ }^{6-12}$ which can provide more controllable architecture structures of LCB. Several studies have discussed the synthesis of LCB-PP via polymerization reactions between propylene and macromonomers or between propylene and dienes. Although this

\footnotetext{
${ }^{a}$ The Key Laboratory of Polymer Processing Engineering of Ministry of Education, National Engineering Research Center of Novel Equipment for Polymer Processing, South China University of Technology, Guangzhou 510640, China. E-mail: xcyin@ scut.edu.cn; Tel: $+86-20-8711-2422$

${ }^{b}$ School of Mechanical and Automobile Engineering, Hubei University of Arts and Science, Xiangyang, 441053 China
}

method was more controllable, it required sophisticated monomers, specialized catalysts and additional purification steps thus making scale-up processes challenging.

Two other methods to introduce LCB onto PP backbone are irradiation techniques and post-reactor chemical modifications. In many reports, irradiation techniques, such as electron beam (EB) ${ }^{13-16}$ and gamma ray $\left(\gamma\right.$-ray) irradiation, ${ }^{17-20}$ have been chosen to modify structure and performance of PP for the production of LCB-PP with improved melt strength. ${ }^{13,14}$ In order to suppress the extent of chain scission, irradiation is carried out in inert environments with the aid of multi-functional monomers. Dietmar et l. $^{20}$ had compared the effects of electron-beam and gamma-irradiation on molecular structures and rheological properties of polypropylene. They found that gamma-irradiation technique led to higher degree of LCB and electron-beam irradiation induced less but probably longer LCBs with a star-like structure at smaller irradiation doses and a treelike structure at higher doses. The major drawback of this method is the presence of undesired side reactions (crosslinking and chain scission). Regardless of its challenges, the irradiation technique has been proved to be a feasible way and adopted to prepare commercial LCB-PP in the market.

LCB-PP can alternatively be synthesized by reactive extrusion of molten state PP in the presence of peroxide chemicals. ${ }^{21-24}$ During extrusion, radicals produced from peroxide chemicals abstract hydrogen atoms from PP backbone to form PP macroradicals. Due to the chemical nature of PP, it has a tendency to undergo $\beta$-scission, which competes with grafting and 
cross-linking reaction, especially while peroxide is added in reaction system only. ${ }^{25}$ To keep under control degradation and provide LCB with controlled and optimized macromolecular structure/architecture, it is necessary to use co-agents able to fasten react with macroradicals (inhibiting the chain breaking and providing coupling between them). ${ }^{26-30}$ However, to our knowledge, solid or liquid organic peroxides were either premixed with the polymer or injected into PP matrix during melt extrusion. Peroxides reach their decomposition temperature prior to effective mixing with $\mathrm{PP}$, thus causing an excessive and non-homogenous degradation in PP.

As we all know, ozone is an advanced form of oxygen. Ozone has been used as an aggressive agent to degrade rubbers and plastics by attacking main chain backbones. ${ }^{31,32}$ Furthermore, ozone has been used to induce polar groups on the polymer surface through oxidation or grafting reactions..$^{33-37}$

Previously, we used ozone to initiate molecular breakdown and oxidization of PP macromolecules with highly efficiency during extrusion process. ${ }^{38}$ In this work, the production of LCBPP via in situ ozonolysis during reactive extrusion process in the presence of multi-functional monomers (trimethylolpropane triacrylate, TMPTA) is reported. The introduction of ozone gas as initiator into molten PP extrusion process can initiate macroradicals at the gas/melt interface. TMPTA serve as linkages between two or more PP radicals leading to the formation of branched structures. Excessive ozone flowed into ozone destructor and decomposed. Neither byproducts nor residual peroxide was left in PP matrix. Conditions of this synthesis process such as polymer flow rate, reaction temperature and TMPTA concentration is discussed.

\section{Materials and methods}

\subsection{Materials}

A commercial polypropylene homopolymer (PP T30s) from Maoming Petrochemical Corporation was used in this study, which has a melt flow index of $3.5 \mathrm{~g} / 10 \mathrm{~min}\left(230^{\circ} \mathrm{C}, 2.16 \mathrm{~kg}\right)$. Ozone was generated electrochemically using dry air by an ozone generator (OZ-3G) from Guangzhou Chuanghuan Ozone Electric Appliance Co., Ltd. Trimethylolpropane triacrylate (TMPTA) containing 600 ppm monomethyl ether hydroquinone as inhibitor was purchased from Sigma-Aldrich Company.

\subsection{Sample preparation}

A co-rotating twin-screw extruder TSE 25/40 with $L / D=40 / 1$ (eight heating zones) from the Brabender® $\mathrm{GmbH} \&$ Co. KG was employed in this experiment. The same screw configuration and ozone generator had previously been used for production of controlled rheology PP in our last report. ${ }^{38}$ A left-handed screw element was set just before the reaction zone for PP melt pressure building-up and uniformity improvement. It was proved to be effective for ozone to induce PP degradation reaction. Ozone is produced from oxygen as a direct result of electrical discharge. The required amount of TMPTA was first dissolved in acetone before mixing with the PP pellets. Then PP pellets were fed at the first barrel zone and heated to molten state through the extruder.
Ozone flowed into the seventh barrel zone where PP was completely melted. The actual length of the ozone reaction zone is about $50 \mathrm{~mm}$. The ozone concentration used throughout this experiment was kept at $6 \mathrm{mg} \mathrm{L}{ }^{-1}$ and the flow rate was $3 \mathrm{~L} \mathrm{~min}^{-1}$.

Experiments were carried out by three different temperatures $\left(190{ }^{\circ} \mathrm{C}, 210{ }^{\circ} \mathrm{C}\right.$ and $\left.230{ }^{\circ} \mathrm{C}\right)$ for reaction zone and three different polymer throughput $\left(7.3 \mathrm{~g} \mathrm{~min}^{-1}, 17.3 \mathrm{~g} \mathrm{~min}^{-1}, 26.8 \mathrm{~g}\right.$ $\left.\min ^{-1}\right)$, respectively. Four different TMPTA concentrations $(0,0.4,0.6,0.8 \mathrm{wt} \%)$ were chosen in this experiment. PP samples were identified as PP with a number corresponding to the TMPTA content. For example, PP-T0 was the samples with 0 wt\% TMPTA and PP-T8 was the samples with 0.8 wt $\%$ TMPTA.

\subsection{Characterization}

Melt flow index (MFI) of PP extrudates was measured using a MP993a melt indexer (Tinius Olsen Testing Machine Co. Inc), according to ASTM Standard 1238 at $230{ }^{\circ} \mathrm{C} / 2.16 \mathrm{~kg}$.

FT-IR spectra of PP extrudates were obtained using a Fouriertransform infrared spectrometer (Nicolet Company, Model Nexus 670). Prior to pursuing FT-IR analysis, about $1 \mathrm{~g}$ sample of PP extrudates was dissolved into refluxing xylene $(250 \mathrm{ml})$ and then precipitated into a large excess of acetone. The polymer was then washed with acetone for three times and dried under vacuum at $100{ }^{\circ} \mathrm{C}$ overnight. In this way, the cleaned polymers were rid of unreacted and homopolymerized TMPTA which are soluble in acetone. The purified samples were hot-pressed into films which are ready to analyze with the FT-IR spectrometer.

Molecular data was measured by high-temperature sizeexclusive chromatography (SEC) coupled with a multiangle laser light scattering (MALLS) detector, a differential refractive index (RI) detector and a capillary viscometer detector (CV). The SEC experiments were carried out using the apparatus PL-GPC 220 (Polymer Laboratories) at $150{ }^{\circ} \mathrm{C}$. The columns set were $3 \times$ PLgel $10 \mu \mathrm{m}$ MIXED-B LS $300 \times 7.5 \mathrm{~mm}$. The eluent solvent was TCB (1,2,4-trichlorobenzene) stabilized with $0.0125 \%$ BHT. The solvent was injected to the system and the elution flow rate

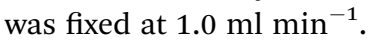

Physica MCR302 rheometer from Anton Paar equipped with a CTD620 convection oven was used to measure the dynamic rheological behaviour of PP samples. Samples obtained from the extruder were compression-molded at $200{ }^{\circ} \mathrm{C}$ for $6 \mathrm{~min}$, and disk-shaped specimens with a thickness of $1 \mathrm{~mm}$ and a diameter of $25 \mathrm{~mm}$ were prepared. The measurements of the dynamic rheological properties were performed with a parallelplate fixture (diameter $=25 \mathrm{~mm}$ ), with a gap distance of $1 \mathrm{~mm}$, and the strain was kept at $2 \%$ to ensure linear viscoelastic response. The frequency range was $0.0628-628 \mathrm{rad} \mathrm{s}^{-1}$, and the temperature was $200{ }^{\circ} \mathrm{C}$. Tests were run under nitrogen purge at

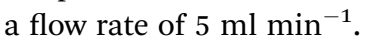

Elongational rheology was taken on a Physica MCR302 rheometer from Anton Paar at $190{ }^{\circ} \mathrm{C}$. The sample sheets were cut into pieces with a width of $10 \mathrm{~mm}$ and a length of $17 \mathrm{~mm}$ for the measurements. The elongational rate was $0.05,0.1,0.3$ and $0.5 \mathrm{~s}^{-1}$ respectively. Before each measurement, a pre-stretch for $9 \mathrm{~s}$ was performed to ensure that no slipping occurred between the sample and the fixture. 
The amount of the insoluble portion (macrogel) in the samples was determined by Soxhlet extraction in refluxing xylene for 24 hours. TMPTA copolymers were removed via acetone. Under the experimental conditions used, the modified PPs are gel-free.

\section{Results and discussions}

As discussed previously in literature, ozone is prone to attack amorphous polypropylene to cause main chain break through a radical mechanism..$^{30,35}$ In this work, PP extruded at molten state had more amorphous molecules than solid PP. The $\beta$-chain scission of PP molecules occurred with formation of an olefin end group at one chain end. If TMPTA were added, PP macroradicals were expected to form long-chain branches and suppress the degradation reaction. The chemical reaction mechanism of the reactive extrusion process might be as shown in Fig. 1. Although acrylate polymers are also prone to betascission, the proposed mechanisms below are supposed to be the major reactions. ${ }^{47-49}$

The absolute molar mass of long-chain branched polymers can be determined simultaneously by SEC-MALS which combines of size-exclusion chromatography (SEC) with multiangle light scattering (MALS) techniques. The parameters of average molar mass and molar mass distributions are given in Table 1. Fig. 2 shows the MWD curves of these samples. Compared to the original PP sample, the molar masses of PP-T0 sample decreased. The dispersity factor $M_{\mathrm{m}} / M_{\mathrm{n}}$ (mass average molar mass to number average molar mass) is reduced from 3.02 (original PP) to 2.51 (PP-T0). This fact leads to the conclusion that the chain scission process is predominant for this ozone induced reaction. With addition of TMPTA into this ozone-induced reactive extrusion, a recovery in average molar mass ( $M_{\mathrm{m}}$ and $M_{\mathrm{n}}$ in Table 1$)$ and molar mass distribution of PP was observed (2.87 for PP-T8). These results indicated a change from degradation reaction to the competitive branching reaction.

The dependence of the root-mean-square radius of gyration on the molar mass for the original PP and modified samples with TMPTA was shown in Fig. 3. Based on the fact that branched polymer chains in solution are denser and have lower root-mean-square radius of gyration than linear chains of the same molecular weight, these plots could provide information about LCB distributions. The slope of 0.59 determined for linear

(1)

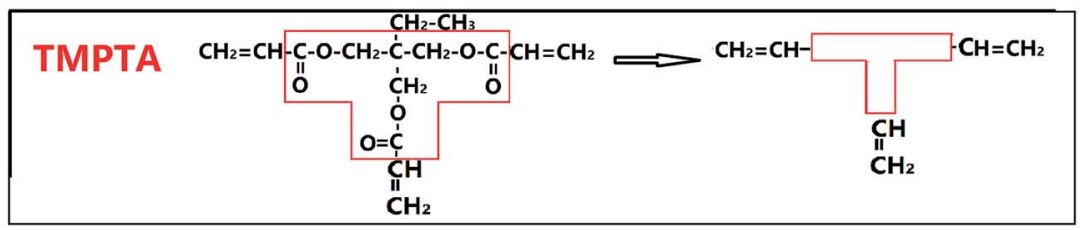

(2)

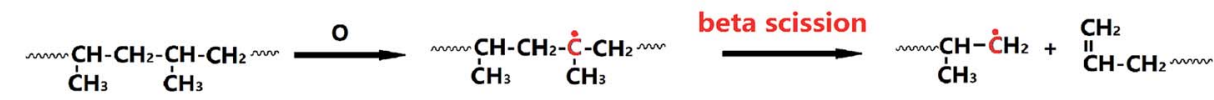

(3)

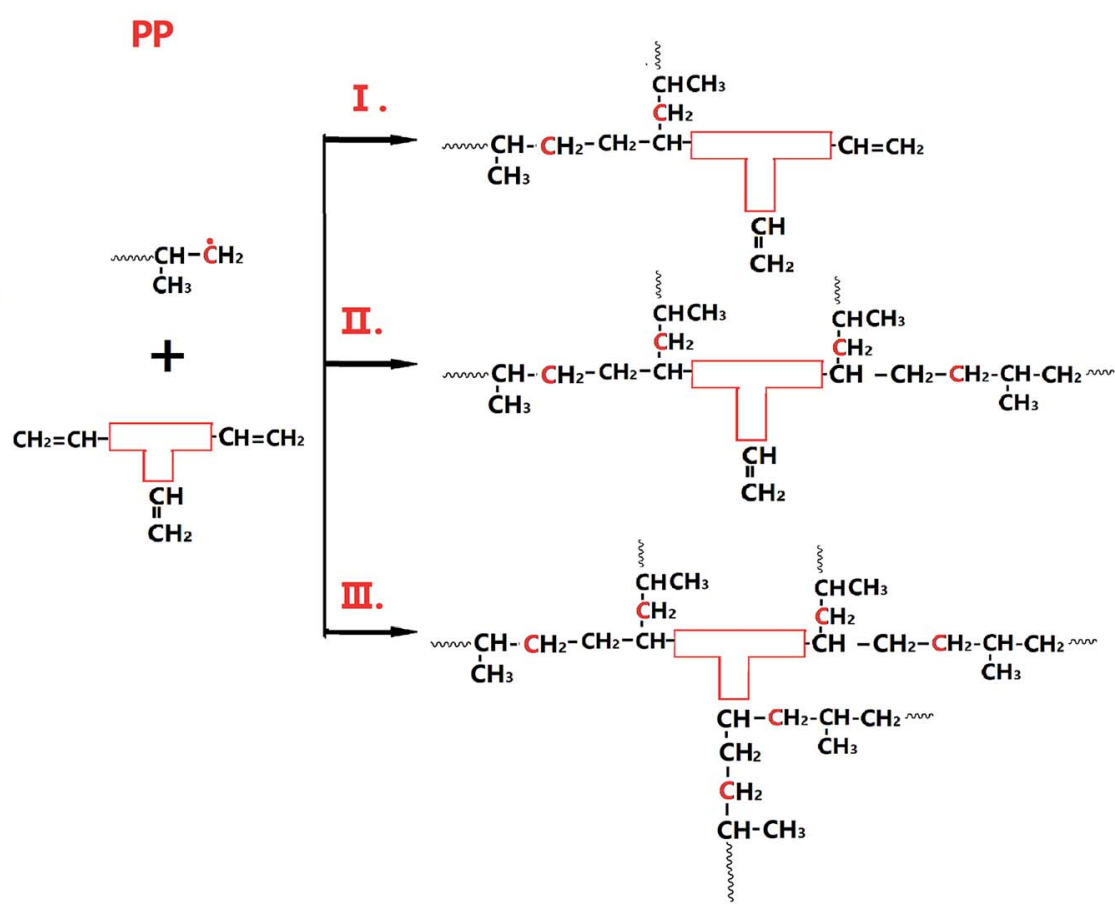

Fig. 1 Possible schematic of PP branching reaction mechanism induced by ozonation in the presence of TMPTA. 
Table 1 Molecular parameters and rheological parameters of original PP and extruded PPs with different TMPTA concentrations (reaction temperature $210{ }^{\circ} \mathrm{C}$ polymer throughput $\left.=26.8 \mathrm{~g} \mathrm{~min}^{-1}\right)^{a}$

\begin{tabular}{|c|c|c|c|c|c|c|c|c|}
\hline Samples & $M_{\mathrm{n}}\left(\mathrm{kg} \mathrm{mol}^{-1}\right)$ & $M_{\mathrm{m}}\left(\mathrm{kg} \mathrm{mol}{ }^{-1}\right)$ & $M_{\mathrm{m}} / M_{\mathrm{n}}$ & $\operatorname{MFI}(\mathrm{g} / 10 \mathrm{~min})$ & $\eta_{\mathrm{o}}(\mathrm{Pa} \mathrm{s})$ & $\lambda(\mathrm{s})$ & Terminal slope of $G^{\prime}$ & LCBD \\
\hline Original PP & 123.7 & 373.3 & 3.02 & 3.50 & 2583 & 0.016 & 1.55 & n.d. \\
\hline PP-T0 & 115.6 & 290.1 & 2.51 & 12.05 & 1118 & 0.006 & 1.51 & n.d. \\
\hline PP-T6 & 135.5 & 369.9 & 2.73 & 4.04 & 4651 & 0.017 & 1.02 & 0.474 \\
\hline PP-T8 & 136.5 & 391.7 & 2.87 & 1.86 & 8935 & 0.019 & 0.85 & 0.495 \\
\hline
\end{tabular}

${ }^{a}$ n.d.: not detected. $\lambda$ is the characteristic relaxation time determined from rheological measurement. LCBD is the number of LCB per 1000 monomers calculated from SEC measurements.

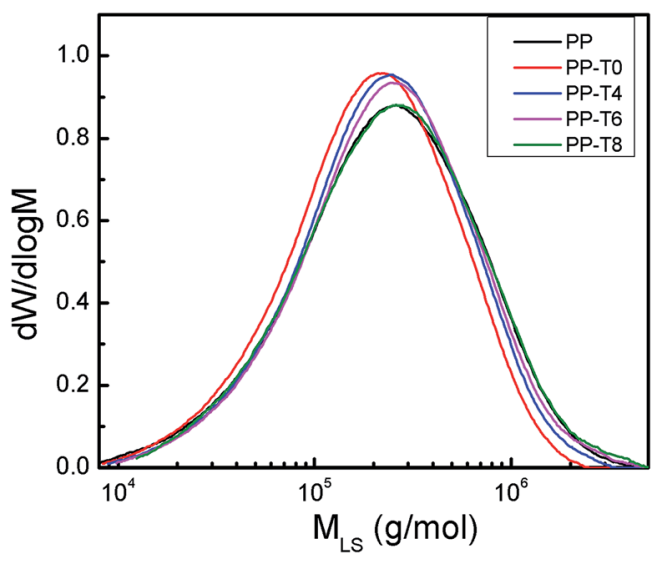

Fig. 2 Molar mass distribution for the original PP and modified samples with different TMPTA concentrations (reaction temperature $210{ }^{\circ} \mathrm{C}$ polymer throughput $=26.8 \mathrm{~g} \mathrm{~min}^{-1}$ ).

PP was found to be higher than the theoretical value of 0.5 for theta solution since the temperature and solvent used for the experiments did not provide theta conditions. The two plots of original PP and PP-T0 were very close to the line and deviated a little in high molecular weight region due to experimental error. In high molecular weight region, the negative deviation of the slope from linear behavior in modified PP plots appeared.

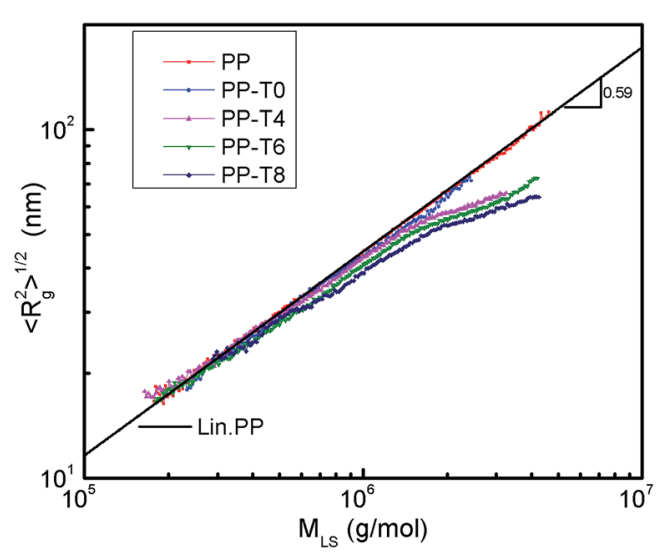

Fig. 3 Root-mean-square radius of gyration $v s$. the molar mass of original linear PP and modified PP samples with different concentrations of TMPTA ( $150{ }^{\circ} \mathrm{C}$ and TCB as solvent).
More LCBs formed on PP chains, more obvious deviation could be seen. This proved that LCB structures were formed on PP molecules during this process with aid of TMPTA. The number of LCB per 1000 monomers (long chain branch density, LCBD) based on Zimm-Stockmayer parameters was also listed in Table 1 , in which we could see the LCBD of branched PP samples increased with the concentration of TMPTA.

MFI is inversely related to viscosity and most of the MFI conditions are at much lower shear than those prevailing in commercial processing. Low molecular weight fractions and long chain branching fractions of PP can apparently influence the flow properties. The MFI of original PP and extruded PPs with different TMPTA concentrations were also listed in Table 1. MFI of PP-T0 is higher than that of the original PP (3.5 g/10 min), proving a degradation reaction of PP induced by ozone. However, MFI decreases rapidly with increasing TMPTA concentration in PP matrix, which indicates the main chain scission might be suppressed and a recovery in molar mass of PP molecules was observed. When TMPTA concentration was excess $0.6 \%$ wt, MFI of modified PP remained relatively constant.

The IR spectra of all samples were shown in Fig. 4. Compared with the FTIR spectrum of PP-T0, new absorption band at about $1734 \mathrm{~cm}^{-1}$ appeared in the spectrum of PP samples with TMPTA addition, which is attributed to the stretching vibration of the carboxyl group of ester in the TMPTA molecule. These results

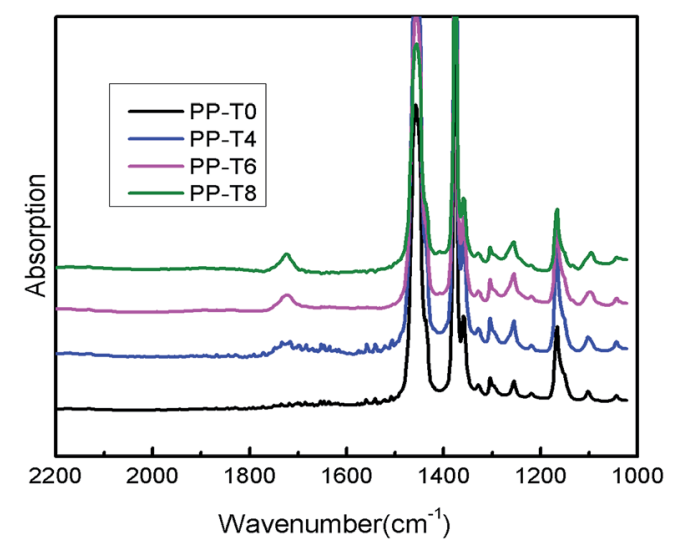

Fig. 4 FT-IR of extruded PPs with different TMPTA concentrations (reaction temperature $210^{\circ} \mathrm{C}$ polymer throughput $=26.8 \mathrm{~g} \mathrm{~min}^{-1}$ ). 
indicate that TMPTA has been grafted onto the PP backbone by this ozonolysis process during the reactive extrusion. In addition, the intensity of the bands at $1734 \mathrm{~cm}^{-1}$ increases with TMPTA concentration, suggesting that more TMPTA molecules were grafted onto PP backbones and would favor macroradicals recombination.

Linear viscoelastic measurement has been proved to be a reliable method for verification of existence of LCB on polymeric chains. The presence of very low amounts of LCB can change the zero-shear viscosity $\left(\eta_{0}\right)$ and the degree of shear thinning, as compared to the linear polymers with similar molecular weight. ${ }^{39,40}$ The complex viscosity curves of the modified PPs with different TMPTA concentration as a function of angular frequency are shown in Fig. 5. Zero shear viscosity $\eta_{0}$ of PP samples could be obtained by quantificationally fitting the melt complex viscosity with the Carreau-Yasuda model described below:

$$
\eta^{*}(\omega)=\eta_{0}\left[1+(\lambda \omega)^{m}\right]^{\frac{n-1}{m}}
$$

where $\lambda$ is the characteristic relaxation time, $m$ and $n$ are exponents. The evaluated data from this fitting equation were listed in Table 1.

For ozone-degraded PP sample, we could see that the complex viscosities decreased over the entire frequency range compared with the original PP. The transition zone from Newtonian-plateau to shear-thinning regime shifted to higher frequency, and Newtonian-zone was broader than original PP samples. At low frequency, the zero-shear viscosity of original $\mathrm{PP}$ is $2583 \mathrm{~Pa} \mathrm{~s}$ and it reached a lower value of $1118 \mathrm{~Pa}$ s for the ozone-degraded PP-T0 sample. For samples with addition of TMPTA, the zero-shear viscosity had an obvious increase with the concentration of TMPTA increasing in PP matrix. The zeroshear viscosity of PP-T4, PP-T6 and PP-T8 exceeded the original PP because a large number of LCBs were formed on the backbone of PP and the chain scission reaction was suppressed. Furthermore, the transition from Newtonian-plateau to

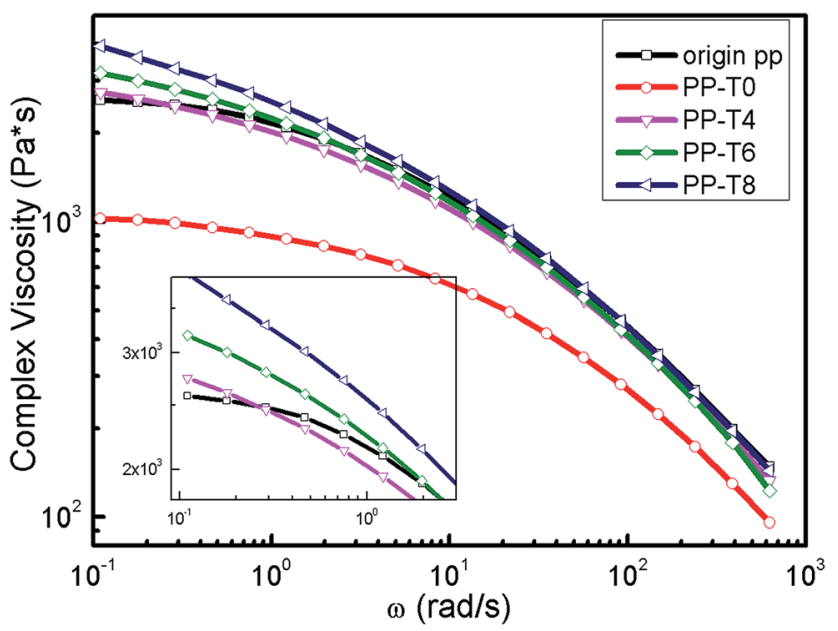

Fig. 5 Complex viscosity vs. angle frequency of original PP and extruded PPs with different TMPTA concentrations (reaction temperature $210^{\circ} \mathrm{C}$ polymer throughput $=26.8 \mathrm{~g} \mathrm{~min}^{-1}$ ). shear-thinning regime is shifted to lower frequency. A broader transition zone between the Newtonian behavior and the powerlaw region is observed for the branched polypropylenes, which is typical of long-chain branched polymers. ${ }^{41}$ In addition, chain relaxation is the main parameter governing this melt property. The evaluated characteristic relaxation time $(\lambda)$ increased with the chain branching level from $0.006 \mathrm{~s}$ for PP-T0 to $0.019 \mathrm{~s}$ for PP-T8 (Table 1) because of the existence of long chain branching structure.

Besides zero-shear viscosity, the storage modulus and the loss angle are even more sensitive to LCB. $^{\mathbf{4 2 , 4 3}}$ In the terminal zone, where only the longest relaxation times contribute to the viscoelastic behavior, $G^{\prime}$ and $G^{\prime \prime}$ of linear polymers follow the well-known frequency dependence, i.e., $G^{\prime} \propto \omega^{2}$ and $G^{\prime \prime} \propto \omega$. In Fig. 6, we can see that original PP and PP-T0 exhibited the typical terminal behavior of linear polymers. With the addition of TMPTA, the $G^{\prime}$ value of LCB-PPs at low frequency (terminal zone) increased gradually and the relation of $G^{\prime} \propto \omega^{2}$ deviated from the terminal behavior. The terminal slope of $G^{\prime}$ decreased from 1.51 of PP-T0 to 0.85 of PP-T8 (Table 1). A second elastic plateau of $G^{\prime}$ for LCB-PPs appears in responding to an increase of the terminal relaxation time, which can be ascribed to the long chain branches formed from this ozone-induced reactive extrusion.

The effect of reaction temperature and polymer throughput on the complex viscosity curves of LCB-PPs are shown in Fig. 7 and 8. The complex viscosity of LCB-PPs decreased with the reaction temperature increased and polymer throughput reduced, indicating that the degradation prevail over branching reactions in high reaction temperature and low polymer throughput. It is reasonably explained that high reaction temperature will induce more $\beta$-scission, and lower throughput will lead to relatively longer degradation time and thinner melt stream in extruder which are in favor of chain scission reaction.

The non-terminal behavior of LCB-PPs can also be illustrated in Cole-Cole plots $\left(\eta^{\prime \prime}-\eta^{\prime}\right.$, Fig. 9), showing the imaginary part $\eta^{\prime \prime}$ of complex viscosity as a function of the real part $\eta^{\prime}$ for the extruded PPs with different TMPTA concentrations. The differences of these samples are even more evident. For the PP-T0, the

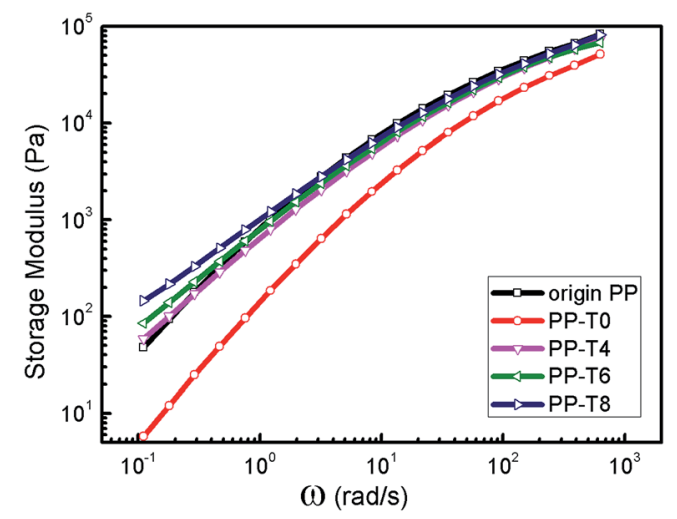

Fig. 6 Storage modulus as functions of angular frequency for original PP and extruded PPs with different TMPTA concentrations (reaction temperature $210{ }^{\circ} \mathrm{C}$ polymer throughput $=26.8 \mathrm{~g} \mathrm{~min}^{-1}$ ). 


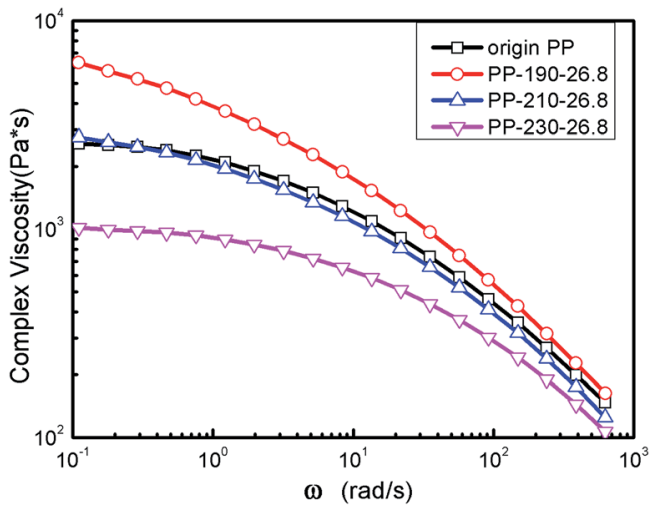

Fig. 7 The effect of reaction temperature on the complex viscosity of LCB-PP with TMPTA (polymer throughput $26.8 \mathrm{~g} \mathrm{~min}^{-1}$. TMPTA concentration $0.4 \% \mathrm{wt}$, reactive temperature $190{ }^{\circ} \mathrm{C}, 210^{\circ} \mathrm{C}, 230{ }^{\circ} \mathrm{C}$, respectively).

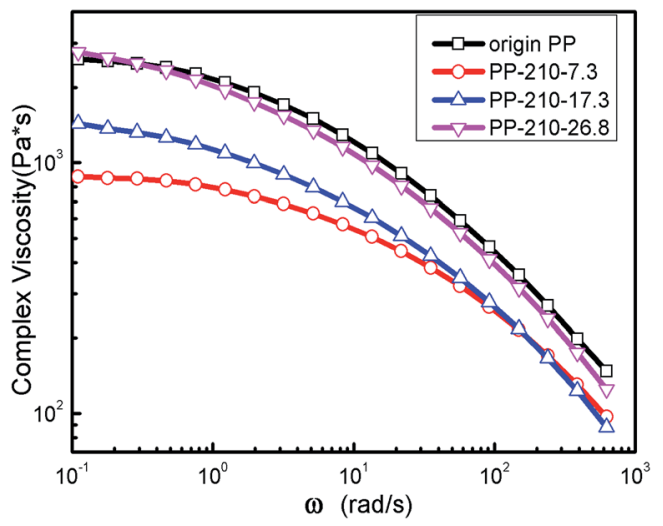

Fig. 8 The effect of polymer throughput on the complex viscosity of LCB-PP with TMPTA (reactive temperature $210{ }^{\circ} \mathrm{C}$, TMPTA concentration $0.4 \% \mathrm{wt}$, polymer throughput $7.3,17.3$ and $26.8 \mathrm{~g} \mathrm{~min}^{-1}$, respectively).

Cole-Cole plots were close to a semicircle which was a typical linear molecular structure. For other PP samples with addition of TMPTA, the Cole-Cole plots were higher than the PP-T0 and showed more evident upturning when the amount of TMPTA

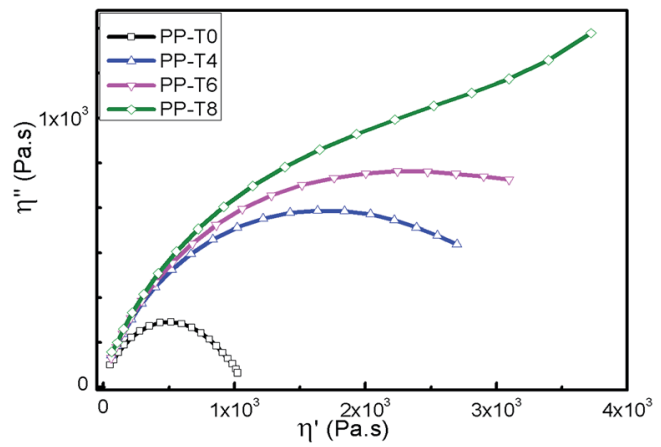

Fig. 9 Cole-Cole plot of extruded PPs with different TMPTA concentrations (reaction temperature $210{ }^{\circ} \mathrm{C}$ polymer throughput $=$ $\left.26.8 \mathrm{~g} \mathrm{~min}^{-1}\right)$.

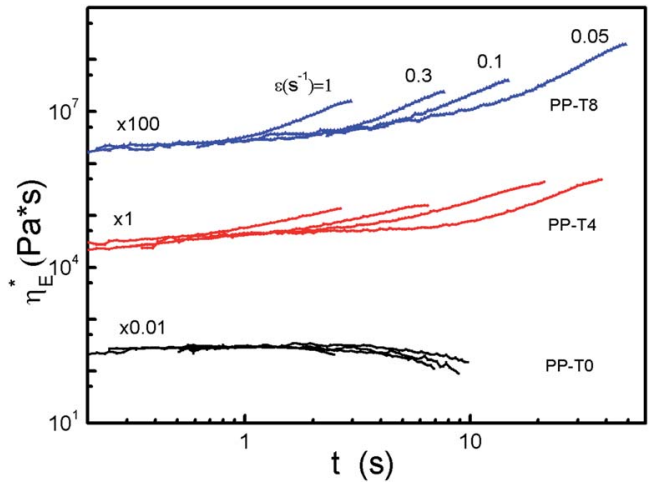

Fig. 10 Transient elongational viscosity $\eta^{*}(t)$ as a function of time at different Hencky strain rates for the extruded PPs with different TMPTA concentrations at $190{ }^{\circ} \mathrm{C}$ (reaction temperature $210{ }^{\circ} \mathrm{C}$, polymer throughput $=26.8 \mathrm{~g} \mathrm{~min}^{-1}$ ).

was higher than $0.8 \mathrm{wt} \%$. Similar results were also reported elsewhere. ${ }^{44-46}$

Uniaxial elongation flow experiments at different elongational rates were performed on the modified PP series. The elongation viscosities were presented in Fig. 10 at various constant Hencky strain rates. The Hencky strain, also called logarithmic strain, is a favored measure of strain due to its remarkable properties at large deformations. As expected, no strain hardening was found for the PP-T0 sample within the experimental accuracy. In contrast, apparent strain hardening deviations were shown for samples with higher TMPTA concentrations.

In this work, the linear chain of original PP can break through $\beta$-scission reaction due to the attack of ozone. In the presence of TMPTA, these newly formed fragments recombine to form linear or long chain branching molecules. Therefore, the product of modified PP with TMPTA in this experiment would be a mixture of linear molecules and branched molecules. These two different molecular structures show different relaxation mechanisms. To illustrate this point, the continuous relaxation spectrum of all samples were calculated (Fig. 11)

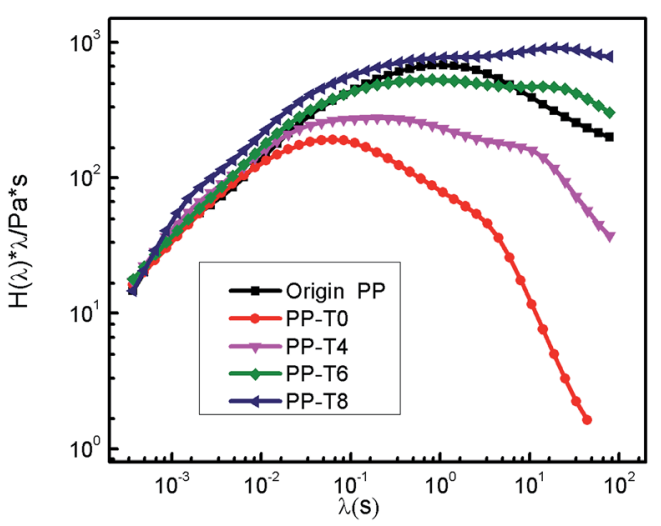

Fig. 11 Weighted relaxation spectrum of original PP and extruded PPs with different TMPTA concentrations (reaction temperature $210^{\circ} \mathrm{C}$ polymer throughput $=26.8 \mathrm{~g} \mathrm{~min}^{-1}$ ). 
from the dynamic modulus. ${ }^{\mathbf{5 0 , 5 1}}$ As seen in Fig. 11, the shorttime peak in the spectrum of degraded PP sample is due to the reptation of the linear chains. However, besides the linear one, an additional longer characteristic relaxation time appeared in LCB-PP relaxation spectra which are a cumulative effect of the relaxations of molecules with various branching chains structures. In addition, more broadening relaxation spectrum can be seen in the PP sample with more amount of TMPTA in the reaction. This result is in agreement with previous works by Tian et al. who obtained LCB-PP using PETA as the co-agent. ${ }^{52}$

\section{Conclusions}

We report a novel synthesis method for the production of LCBPP via in situ ozonolysis during reactive extrusion process in the presence of co-agent trimethylolpropane triacrylate (TMPTA). Ozonation of molten PP molecules could produce free radicals efficiently so as to induce chain scission reactions of PP during extrusion. LCB structures in PP molecules could be formed when TMTPA was added. The amount of LCB produced by this method has an increase trend with more TMPTA concentrations. Long-chain branching PP showed a significant influence on the viscoelastic properties of the melts. Compared to the original polypropylene, a significant increase of the zero shear viscosities of the LCB-PP samples was observed. The onset of the shear thinning region started at lower frequency and the Newtonian behavior disappeared gradually with the increase of TMPTA concentration. The LCB-PP samples showed a distinct strain hardening behavior in elongational flow with changing strain rate dependence. The continuous weight relaxation spectrum showed an additional longer relaxation time in LCBPP spectrum, which indicated that the product was a mixture of linear molecules and branched molecules.

\section{Acknowledgements}

Financial support from the National Nature Science Foundation of China (51435005, 51403059), the Special-funded Program on National Key Scientific Instruments and Equipment Development of China (2012YQ230043) and Fundamental Research Funds for the Central Universities of China (2015ZZ132 and 2015ZZ067), are gratefully acknowledged.

\section{References}

1 H. C. Lau, S. N. Bhattacharya and G. J. Field, Melt strength of polypropylene: its relevance to thermoforming, Polym. Eng. Sci., 1998, 38(11), 1915-1923.

2 B. Lu and T. C. Chung, Synthesis of long: chain branched polypropylene with relatively well-defined molecular structure, Macromolecules, 1999, 32(25), 8678-8680.

3 W. Q. Weng, E. J. Markel and A. H. Dekmezian, Synthesis of vinyl-terminated isotactic poly(propylene), Macromol. Rapid Commun., 2000, 21(16), 1103-1107.
4 W. Q. Weng, W. G. Hu, A. H. Dekmezian and C. J. Ruff, Long chain branched isotactic polypropylene, Macromolecules, 2002, 35(10), 3838-3843.

5 H. Y. Wang, X. T. Hu, Z. Y. Li, J. J. Yi and J. Y. Dong, Preparation and characterization of high melt strength polypropylene, Process. Chem., 2007, 19(6), 932-958.

6 Z. Ye, F. Alobaidi and S. Zhu, Synthesis of branched polypropylene with isotactic backbone and atactic side chains by binary iron and zirconium single-site catalysts, J. Polym. Sci., Part A: Polym. Chem., 2003, 41(8), 1152-1159.

7 J. A. Langston, R. H. Colby and T. C. M. Chung, Synthesis and characterization of long chain branched isotactic polypropylene via metallocene catalyst and T-reagent, Macromolecules, 2007, 40(8), 2712-2720.

$8 \mathrm{~W}$. Kaminsky and S. Rulhoff, Long-chain branched polypropylenes obtained by copolymerization with use of metallocene catalysts, Polymer, 2008, 53(5), 339-344.

9 J. Langston, J. Y. Dong and T. C. Chung, One-pot process of preparing long chain branched polypropylene using C-2symmetric metallocene complex and a " $\mathrm{T}$ " reagent, Macromolecules, 2005, 38(14), 5849-5853.

10 Z. B. Ye, F. AlObaidi and S. P. Zhu, Synthesis and rheological properties of long-chain-branched isotactic polypropylenes prepared by copolymerization of propylene and nonconjugated dienes, Ind. Eng. Chem. Res., 2004, 43, 2860-2870.

11 B. D. Zhu, W. C. Dong, J. Wang, J. Song and Q. Dong, Modification of polypropylene via the free-radical grafting ternary monomer in water suspension systems, J. Appl. Polym. Sci., 2012, 126, 1844-1851.

12 C. H. Zhang, H. Niu and J. Y. Dong, Fabrication of long chain branched polypropylene using click chemistry, Polym. Bull., 2012, 68, 949-959.

13 B. Krause, M. Stephan, S. Volkland, D. Voigt, L. Haussler and H. Dorschner, Long-chain branching of polypropylene by electron-beam irradiation in the molten state, J. Appl. Polym. Sci., 2006, 99, 260-265.

14 F. Yoshii, F. Makuuchi and S. Kikukawa, High-melt-strength polypropylene with electron beam irradiation in the presence of polyfunctional monomers, J. Appl. Polym. Sci., 1996, 60, 617-623.

15 B. Krause, D. Voigt, L. Haussler, D. Auhl and H. Munstedt, Characterization of electron beam irradiated polypropylene: influence of irradiation temperature on molecular and rheological properties, J. Appl. Polym. Sci., 2006, 100, 2770-2780.

16 D. Auhl, J. Stange, H. Munstedt, B. Krause, D. Voigt, A. Lederer, U. Lappan and K. Lunkwitz, Long-chain branched polypropylenes by electron beam irradiation and their rheological properties, Macromolecules, 2004, 37, 9465-9472.

17 J. Lacoste, D. Vaillant and D. J. Carlsson, Gamma-, photo-, and thermally-initiated oxidation of isotactic polypropylene, J. Polym. Sci., Part A: Polym. Chem., 1993, 31, 715-722.

18 A. B. Lugao, B. Hutzler, T. Ojeda, S. Tokumoto, R. Siemens and K. Makuuchi, Reaction mechanism and rheological 
properties of polypropylene irradiated under various atmospheres, Radiat. Phys. Chem., 2000, 57, 389-392.

19 A. B. Lugao, H. Otaguro, D. F. Parra, A. Yoshiga, L. F. C. P. Lima, B. W. H. Artel and S. Liberman, Review on the production process and uses of controlled rheology polypropylene-Gamma radiation versus electron beam processing, Radiat. Phys. Chem., 2007, 76, 1688-1690.

20 A. Dietmar, S. Jens and M. Helmut, Comparison of molecular structure and rheological properties of electron-beam- and gamma-irradiated polypropylene, Macromolecules, 2012, 45, 2057-2065.

21 Z. J. Zhang, D. Wan, H. P. Xing, Z. J. Zhang, H. Y. Tan, L. Wang, J. Zheng, Y. J. An and T. Tang, A new grafting monomer for synthesizing long chain branched polypropylene through melt radical reaction, Polymer, 2012, 53, 121-129.

22 F. Yoshii, K. Makuuchi and S. Kikukawa, High melt strength polypropylene with electron beam irradiation in the presence of polyfunctional monomers, J. Appl. Polym. Sci., 1996, 60, 617-623.

23 R. P. Lagendijk, A. H. Hogt, A. Buijtenhuijs and A. D. Gotsis, Peroxydicarbonate modification of polypropylene and extensional flow properties, Polymer, 2001, 42, 10035.

24 D. Graebling, Synthesis of Branching Polypropylene by a Reactive Extrusion Process, Macromolecules, 2002, 35, 4602-4610.

25 K. E. Mabrouk, J. S. Parent, B. I. Chaudhary and R. Cong, Chemical modification of PP architecture: strategies for introducing long-chain branching, Polymer, 2009, 50, 5390-5397.

26 S. A. Mousavi, M. Frounchi, S. Dadbin, S. Augier, E. Passaglia and F. Ciardelli, Modification of isotactic polypropylene by the free-radical grafting of 1,1,1-trimethylolpropane trimethacrylate, J. Appl. Polym. Sci., 2007, 104, 950-958.

27 S. Augier, S. Coiai, E. Passaglia, F. Ciardelli, F. Zulli, L. Andreozzi and M. Giordano, Structure and rheology of polypropylene with various architectures prepared by coagent-assisted radical processing, Polym. Int., 2010, 59, 1499-1505.

28 M. F. Diop and J. M. Torkelson, Novel synthesis of branched polypropylene via solid-state shear Pulverization, Polymer, 2015, 60, 77-87.

29 E. Borsig, M. van Duin, A. D. Gotsis and F. Picchioni, Long chain branching on linear polypropylene by solid state reactions, Eur. Polym. J., 2008, 44, 200-212.

30 M. Ratzsch, M. Arnold, E. Borsig, H. Bucka and N. Reichelt, Radical reactions on polypropylene in the solid state, Prog. Polym. Sci., 2002, 27, 1195-1282.

31 G. Meijers and P. Gijsman, Influence of environmental concentrations of ozone on thermo-oxidative degradation of PP, Polym. Degrad. Stab., 2001, 74, 387-391.

32 X. W. Cao, J. Luo, Y. Cao, X. C. Yin, G. J. He, X. F. Peng and B. P. Xu, Structure and properties of deeply oxidized waster rubber crumb through long time ozonization, Polym. Degrad. Stab., 2014, 109, 1-6.

33 M. Michalak, M. Hakkarainen and A. Albertsson, Recycling oxidized model polyethylene powder as a degradation enhancing filler for polyethylene/polycaprolactone blends, ACS Sustainable Chem. Eng., 2016, 4, 129-135.

34 F. Cataldo and M. Omastova, On the ozone degradation of polypyrrole, Polym. Degrad. Stab., 2003, 82, 487-495.

35 F. Cataldo, O. Ursini and G. Angelini, Surface oxidation of rubber crumb with ozone, Polym. Degrad. Stab., 2010, 95, 803.

36 S. G. Hong and C. M. Liao, The surface oxidation of a thermoplastic olefin elastomer under ozone exposure: ATR analysis, Polym. Degrad. Stab., 1995, 49, 437-448.

37 F. Cataldo, E. Lilla and O. Ursini, Surface Reaction of Ozone at High Concentration with Isotactic And Syndiotactic Polypropylene, J. Macromol. Sci., Pure Appl. Chem., 2011, 48, 607-618.

38 G. J. He, T. T. Zheng, D. M. Ke, X. W. Cao, X. C. Yin and B. P. $\mathrm{Xu}$, Impact of rapid ozone degradation on the structure and properties of polypropylene using a reactive extrusion process, RSC Adv., 2015, 55, 44115-44120.

39 A. D. Gotsis, B. L. F. Zeevenhoven and A. H. Hogt, The effect of long chain branching on the processability of polypropylene in thermoforming, Polym. Eng. Sci., 2004, 44, 973-982.

40 A. Malmberg, C. Gabriel, T. Steffl, H. Munstedt and B. Lofgren, Long-chain branching in metallocene-catalyzed polyethylenes investigated by low oscillatory shear and uniaxial extensional rheometry, Macromolecules, 2002, 35, 1038-1048.

41 H. Xu, H. Fang, J. Bai, Y. Zhang and Z. Wang, Preparation and Characterization of High-Melt-Strength Polylactide with Long-Chain Branched Structure through $\gamma$-RadiationInduced Chemical Reactions, Ind. Eng. Chem. Res., 2014, 53, 1150-1159.

42 F. J. Stadler, Detecting very low levels of long-chain branching in metallocene-catalyzed polyethylenes, Rheol. Acta, 2012, 51, 821-840.

43 E. V. Ruymbeke, V. Stéphenne, D. Daoust, R. Godard, R. Keunings and C. Bailly, A sensitive method to detect very low levels of long chain branching from the molar mass distribution and linear viscoelastic response, J. Rheol., 2005, 49, 1503-1520.

44 C. A. Franco, S. Srinivas, D. J. Lohse and P. Brant, Similarities between gelation and long chain branching viscoelastic behavior, Macromolecules, 2001, 34, 3115-3117.

45 S. Z. Li, M. M. Xiao, D. F. Wei, H. N. Xiao, F. Z. Hu and A. N. Zheng, The melt grafting preparation and rheological characterization of long chain branching polypropylene, Polymer, 2009, 50, 6121-6128.

46 G. J. Nam, J. H. Yoo and J. W. Lee, Effect of long-chain branches of polypropylene on rheological properties and foam-extrusion performances, J. Appl. Polym. Sci., 2005, 96, 1793-1800.

47 J. S. Parent, A. Bodsworth, S. S. Sengupta, M. Kontopoulou, B. I. Chaudhary and S. Cousteaus, Structure-rheology relationships of long-chain branched polypropylene: comparative analysis of acrylic and allylic coagent chemistry, Polymer, 2009, 50, 85-94.

48 P. Derboven, P. H. M. Van Steenberge, J. Vandenbergh, M.-F. Reyniers, T. Junkers, D. R. D'hooge and G. B. Marin, 
Improved Livingness and Control over Branching in RAFT Polymerization of Acrylates: Could Microflow Synthesis Make the Difference?, Macromol. Rapid Commun., 2015, 36, 2149-2155.

49 C. Toloza Porras, D. R. D'hooge, M.-F. Reyniers and G. B. Marin, Computer-Aided Optimization of Conditions for Fast and Controlled ICAR ATRP of $n$-Butyl Acrylate, Macromol. Theory Simul., 2013, 22, 136-149.
50 D. J. Lohse, S. T. Milner, L. J. Fetters and M. Xenidou, Welldefined, model long chain branched polyethylene. 2. Melt rheological behavior, Macromolecules, 2002, 35, 3066-3075.

$51 \mathrm{~J}$. Honerkamp and J. Weese, A nonlinear regularization method for the calculation of relaxation spectra, Rheol. Acta, 1993, 32, 65-73.

52 J. H. Tian, W. Yu and C. X. Zhou, The preparation and rheology characterization of long chain branching polypropylene, Polymer, 2006, 47, 7962-7969. 